the place of Study's "Geometrie der Dynamen" in the structure of geometry. Geometric figures have been considered whose tensor representations are: 1. contravariant alternating tensors of valence two; 2 . covariant alternating tensors of valence two; 3. mixed tensors of valence two. Among this set are found the affine ancestors of all of the Study figures. Thus the foundation is laid for the tensor interpretation of all of the figures of the "Geometrie der Dynamen." (Received July 30, 1942.)

\title{
Statistics and Probability
}

\section{L. A. Aroian: The relationship of Fisher's z distribution to Student's $t$ distribution.}

For $n_{1}$ and $n_{2}$ sufficiently large, $W=(1 / \beta)(N /(N+1))^{1 / 2} z$ is distributed as Student's $t$ with $N$ degrees of freedom, $N=n_{1}+n_{2}-1, \beta^{2}=(1 / 2)\left(1 / n_{1}+1 / n_{2}\right)$. If the level of significance is $\alpha$ for Student's distribution, the level of significance for $z$ will be $(N /(N+1))^{1 / 2} \alpha e^{\beta^{2} / 3-5 / 12 N}<\alpha$. As a corollary it follows that the distribution of $z$ approaches normality, $n_{1}, n_{2} \rightarrow \infty$, with mean zero and variance $\beta^{2}$. This simplifies a previous proof of the author. Application of this result is made to finding levels of significance of the $z$ distribution. On the whole R. A. Fisher's formulas for finding such levels, $n_{1}$ and $n_{2}$ large as modified by W. G. Cochran, are superior. The formulas of Fisher-Cochran are compared with the recent formula of E. Paulson. (Received August 1,1942.)

\section{E. J. Gumbel: Graphical controls based on serial numbers.}

The index $m$ of the observed value $x_{m}(m=1,2, \cdots, n)$ is called its serial number (or rank). A value $x$ of the continuous statistical variable defined by a probability $W(x)=\lambda$ is called a grade (for example, the median). The coordination of serial numbers with grades furnishes two graphical methods for comparing the observations and the theory, namely the equiprobability test based on $m=n \lambda$, and the return periods based on $m=n \lambda+1 / 2$. From the distribution of the $m$ th value, determine the most probable serial number $\tilde{m}=n \lambda+\Delta$, where $\Delta$ depends upon the distribution. For a symmetrical distribution, the corrections for two grades defined by $\lambda$ and $1-\lambda$, are $\Delta(1-\lambda)=-\Delta(\lambda)$. For an asymmetrical distribution, calculate the most probable serial number of the mode considered as an $m$ th value. Thus the mode is obtained from the observations, but it is not the most precise $m$ th value. If $m$ is of the order $n / 2$ the distribution of the $m$ th value converges towards a normal distribution with a standard deviation $s(x)=(W(x)(1-W(x)))^{1 / 2} /\left(w(x) n^{1 / 2}\right)$. The intervals $x \mp s(x)$ give controls for the equiprobability test, the step function and the return periods. Besides, the standard error of the $m$ th value leads to the precision of a constant obtained from a grade. (Received July 20, 1942.)

\section{Mark Kac: On the average number of roots of a random alge- braic equation.}

Let $X_{0}+X_{1} x+\cdots+X_{n-1} x^{n-1}=0$ be an algebraic equation whose coefficients $X_{0}, \cdots, X_{n-1}$ are independent random variables having the same normal distribution with density $\pi^{-1 / 2} \exp \left(-u^{2}\right)$. If $N_{n}=N_{n}\left(X_{0}, \cdots, X_{n-1}\right)$ denotes the number of real roots of the equation then the average number of roots (mathematical expectation of $N_{n}$ ) is asymptotically equal to $2 \pi^{-1} \log n$. Moreover, for $n \geqq 2$ the mathematical expectation of $N_{n}$ is not more than $2 \pi^{-1} \log n+14 \pi^{-1}$. This is an improvement of a result of Littlewood and Offord (Journal of the London Mathematical Society, 
vol. 13 (1938), pp. 288-295) who proved that for $n \geqq 2000$ the mathematical expectation of the $N_{n}$ is not more than $25(\log n)^{2}+12 \log n$. (Received July 13,1942.)

285. H. B. Mann and Abraham Wald: On the choice of the number of class intervals in the application of the chi-square test.

The distance of two distribution functions is defined as the 1.u.b. of the absolute value of the difference between the two cumulative distribution functions. Let $C(\Delta)$ be the class of alternatives with distance $\Delta$ from the null-hypothesis. Let $f(N, k, \Delta)$ be the g.l.b. of the power with respect to alternatives in $C(\Delta)$ of the chi-square test with sample size $N$ and $k$ equally probable class intervals. A positive integer $k$ is called best with respect to sample size $N$ if there exists a $\Delta$ such that $f(N, k, \Delta)=1 / 2$ and $f\left(N, k^{\prime}, \Delta\right) \leqq 1 / 2$ for every positive integer $k^{\prime}$. The authors show that $k_{N}=4\left(2(N-1)^{2} / C^{2}\right)^{1 / 5}$, where $1 /(2 \pi)^{1 / 2} \int_{C}^{\infty} e^{-x^{2} / 2} d x$ is equal to the size of the critical region, fulfills approximately the conditions of a best $k$ with $\Delta_{N}=5 / k_{N}-4 / k_{N}^{2}$ as the corresponding value of $\Delta$. The approximation is shown to be satisfactory for $N \geqq 450$ if the 5 per cent level of significance is used and for $N \geqq 300$ if the 1 per cent level is used. (Received July 31, 1942.)

\section{F. E. Satterthwaite: Generalized Poisson distribution.}

In this paper the Poisson distribution is generalized to allow for the assignment of varying weights to a set of events when the number of events follows the Poisson law. The development used brings out the fact that distributions falling in this class do not require that the underlying statistics be homogeneous. The only requirement is that they be independent. Formulas are given for the moments of the generalized distribution as functions of the moments of the underlying distribution of weights. The principles to be observed in the solution of practical problems are outlined. (Received July 30,1942 .)

\section{Henry Scheffé: On the ratio of the variances of two normal populations.}

Let $\theta$ be the above ratio. The two problems considered in this paper are the formulation and comparison of (i) significance tests for the hypothesis $\theta=\theta_{0}$, and (ii) confidence intervals for $\theta$. The paper is divided into two parts; the first is kept on an elementary level and only solutions based on the $F$-distribution are considered. Following various approaches, six tests and corresponding sets of confidence intervals are introduced. It turns out that the limits on the $F$-distribution which yield an unbiased test are the same as those which yield confidence intervals optimum in a certain intuitive sense. The values of these limits are difficult to compute and some numerical data are given to indicate the loss of efficiency in using instead the easily obtained "equal tails" limits. The second part of the paper is concerned with the existence of common best critical regions and type $B_{1}$ regions, and the application of Neyman's theory of confidence intervals. No new tests or confidence intervals not already considered in Part I are obtained, but those previously judged best of a very narrow class are now shown to be best of all those based on similar regions of the same size. (Received July 16, 1942.)

288. Saly R. R. Struik and Miriam van Waters: Modern statistical methods in penology.

In applying statistical methods to penological problems, so far the best known 
studies have considered 100,500, or once in England (to refute Lombroso's theory) 1500 cases. But from the correct statistical standpoint, far more cases are needed to establish a law. Over a period of years, an attempt has been made to use statistical methods in the study of penological problems in the Massachusetts Reformatory for Women, but the results will take on real significance and be conclusive only when similar investigations are made all over the United States. (Received August 1, 1942.)

\section{D. S. Villars: Significance tests for multivariate distributions.}

The observed mean of sets of $m$ variates, each normally and independently distributed, is distributed around the population mean according to a $\chi^{2}$ distribution with $m$ degrees of freedom. The sum of squares of deviations of $n$ observed points from the observed mean is distributed as $\chi^{2}$ with $m(n-1)$ degrees of freedom (not with $n-1)$. A much more powerful test for correlation than that by the correlation coefficient is described, which for bivariate distributions, involves comparisons between $n-1$ and $n-1$ degrees of freedom. This can be extended to $m-1$ tests with $m$ variates. Distribution of distance between two means and distribution of fiducial radius is worked out in detail for two variates. (Received July 30,1942.)

\section{TOPOLOGY}

290. D. W. Hall: On a partial solution of a problem of J. R. Kline.

As a partial solution of a problem of J. R. Kline, the following theorem is established. In order that a compact locally connected continuum $M$ be homeomorphic with a sphere it is necessary and sufficient that it satisfy the following conditions: (a) no two points separate $M$, (b) for every simple closed curve $J$ in $M$ the set $M-J$ has at least two and at most a finite number of components. (Received June 22, 1942.)

\section{W. M. Kincaid: On non-cut sets of locally connected continua.}

This paper is concerned with certain generalizations of the well known result that corresponding to any non-cut point $p$ of a space $S$ which is a locally connected continuum, an arbitrarily small region having a connected complement and containing $p$ can be found. It is shown that any closed non-cut set $P$ of such a space $S$ can be imbedded in the sum $R$ of a finite number of regions (lying in a preassigned $\epsilon$-neighborhood of $P$ ) so chosen that $S-R$ is a locally connected continuum. If, in addition, there exists a family of sets $\mathcal{F}$ no element of which separates $S-P$, then another set $R^{\prime}$, contained in $R$ and having the same properties, can be found such that no element of $\mathcal{F}$ contained in $S-R$ separates $S-R^{\prime}$. If the elements of $\mathcal{F}$ are single points, the sets $R$ and $R^{\prime}$ can be replaced by a single set having the properties of both. Further results are obtained in the special case where $S$ is not separated by any $m$ points. (Received July 24, 1942.)

\section{R. G. Lubben: Mappings of spaces H Fréchet on completely regular spaces.}

Let $T$ be a space $H$ Fréchet, $K$ be the aggregate of all completely regular Hausdorff decompositions (Alexandroff and Hopf, Topologie, p. 70; the space of this decomposition is to be a completely regular Hausdorff space) of $T$ into mutually exclusive point sets, and $\sum$ be the sum of the elements of $K$. If $T \supset M$ and for $G=\bar{G} \subset T-M$ there exists a function which is continuous over $T$, takes on values from zero to unity, and 